\title{
On the Spectrum of Fluctuations in an Effective Field Theory of the Ekpyrotic Universe
}

\author{
Robert Brandenberger ${ }^{1)}$ and Fabio Finelli ${ }^{2)}$ \\ 1) Department of Physics, Brown University, Providence, RI 02912, USA. \\ e-mail: rhb@het.brown.edu \\ 2) Department of Physics, Purdue University, West Lafayette, IN 4790\%, USA. \\ e-mail:fabio@physics.purdue.edu
}

(August 28, 2001)

\begin{abstract}
We consider the four-dimensional effective field theory which has been used in previous studies of perturbations in the Ekpyrotic Universe, and discuss the spectrum of cosmological fluctuations induced on large scales by quantum fluctuations of the bulk brane. By matching cosmological fluctuations on a constant energy density hypersurface we show that the growing mode during the very slow collapsing pre-impact phase couples only to the decaying mode in the expanding postimpact phase, and that hence no scale-invariant spectrum of adiabatic fluctuations is generated. Note that our conclusions may not apply to improved toy models for the Ekpyrotic scenario.
\end{abstract}

\section{INTRODUCTION}

Recently, Khoury et al. [1] suggested a new cosmological scenario which they called the Ekpyrotic Universe, a model motivated by string theory (more precisely on Horava-Witten theory [2]). The authors claim it solves the well-known problems of standard cosmology such as the horizon, flatness and monopole problems (see e.g. [3]) without the need to invoke a period of inflationary expansion (see, however, [4] for an opposing view concerning this claim). In particular, a mechanism which predicts a scale-invariant spectrum of adiabatic density fluctuations, whose magnitude can be adjusted to agree with observations, was suggested.

According to the Ekpyrotic Universe, the Big Bang of standard cosmology is replaced by the collision of two parallel branes, one of which is our visible space-time, in a higher dimensional bulk. In the specific realization studied in detail in 11], the visible space-time is an orbifold fixed plane in a five-dimensional bulk space-time, the fifth dimension being a $S^{1} / Z_{2}$ orbifold whose length is substantially larger than the radius of the internal sixdimensional Calabi-Yau space. The second orbifold fixed plane is the hidden brane. The scenario assumes a cold initial Universe with flat orbifold fixed planes, and is based on the suggestion that a light bulk brane is nucleated near the hidden brane, and attracted via a bulk potential to the visible brane. The impact of the bulk brane on the visible brane generates the heating event of the visible Universe which replaces the Big Bang of standard cosmology $\mathrm{f}$.

\footnotetext{
${ }^{*}$ For a discussion of conceptual issues crucial to the Ekpyrotic Universe scenario, in particular the initial condition problem, the reader is referred to [4].
}

This paper concerns the proposed mechanism of generating a scale-invariant spectrum of adiabatic cosmological fluctuations without the need to invoke inflation if. The suggestion is that quantum fluctuations in the position of a light bulk brane in the five-dimensional bulk of heterotic M-theory lead to a space-dependent time delay in the brane collision which results in density fluctuations on the visible brane. Similar to the case of inflationary cosmology 12, small quantum fluctuations can generate a large time delay, and hence large density fluctuations. For a bulk potential given by a single exponential factor, the spectrum calculated using the methods of [1] (which effectively neglect the metric fluctuations) is scale-invariant.

However, Lyth has recently claimed 13] that the results change in a dramatic way when the metric fluctuations are taken into account, and that the curvature fluctuations produced in the Ekpyrotic Universe are in fact not amplified at all on super-Hubble scales and that the resulting spectrum of curvature fluctuations is a blue spectrum with index $n \simeq 3$ instead of scale-invariant $(n=1)$. Note that a blue spectrum is also obtained in the case of simple models of pre-big-bang cosmology 14 which only include the dilaton field 15. Both Khoury et al. and Lyth use an effective four space-time dimensional model which includes, in addition to the graviton, only a single scalar field (a field which represents the location of the bulk brane in the fifth dimension). Lyth's argument is based on the fact that there is no growth of curvature fluctuations on super-Hubble scales.

\footnotetext{
${ }^{\dagger}$ Note that there are toy models based on topological defects which also produce a scale-invariant spectrum (see e.g. [9.10]), but these alternatives lack a convincing microphysical motivation. There also exist some special backgrounds for which pre-big-bang cosmology generates such a spectrum [11].
} 
In this paper, we use the junction conditions for cosmological fluctuations derived by Hwang and Vishniac 16 and by Deruelle and Mukhanov [17 (see also 18]) junction conditions for cosmological fluctuations to demonstrate that, within the context of the effective field theory used in [1] and [13], the spectrum of curvature fluctuations at late times is blue, and that there is no overall amplification of the amplitude between the times when the perturbation mode exits the Hubble radius during the pre-impact phase and when it re-enters during the postimpact period, the reason being that the growing mode of the pre-impact phase couples exclusively to the decaying mode during the post-impact period. Note that our conclusions are based on a particular four-dimensional effective field theory for the Ekpyrotic scenario and may not apply to improved descriptions.

Note, however, that in the Ekpyrotic Universe there are many matter fields which must be considered, in addition to the scalar field representing the position of the bulk brane. In a followup paper, we will investigate the isocurvature modes of these fields which can be excited by the fluctuations of the bulk brane. These modes would then, in turn, induce a growing adiabatic component. This latter mechanism would have close analogies with the physics which describes the growth of axion fluctuations in inflationary cosmology [19] (see also [20]), or the growth of axion fluctuations in pre-big-bang cosmology [11.

The outline of this paper is as follows. After a review of the version of the Ekpyrotic Universe scenario presented in 11] (Section 2), we discuss (Section 3) the effective field theory considered in [1] and [13] and demonstrate (Section 4), using the matching conditions of Hwang and Vishniac [16] and Deruelle and Mukhanov [17], the absence of an overall amplification of the spectrum of fluctuations. We conclude with a discussion of the severe limitations of any four-dimensional effective field theory description.

\section{REVIEW OF THE EKPYROTIC UNIVERSE SCENARIO}

The Ekpyrotic Universe scenario is not the first attempt to combine string theory and cosmology (see e.g. 21,22 for recent overviews of various approaches to string cosmology). As will be shown below, it bears many analogies, in particular in terms of the analysis of fluctuations, with pre-big-bang cosmology [14] (see e.g. [24] for a recent comprehensive review). Crucial to both scenarios is the role which the dilaton or other scalar fields play in the cosmological scenario. In both cases, the Universe is assumed to start in a cold, symmetric statef, although

\footnotetext{
${ }^{\ddagger}$ There is also a very different approach to string cosmology in which the Universe is assumed to start out in a hot thermal
}

in the case of the pre-big-bang scenario this assumption can be somewhat relaxed 26]. Since in this case the Universe has infinite age, the horizon problem is solved. The assumption of initial symmetry produces a Universe without spatial curvature 8

The basic setup is the following. We start from the action of 11-d supergravity compactified on a $S^{1} / Z_{2}$ orbifold and compactify the theory on a Calabi-Yau threefold [2]. The radius of the orbifold is taken to be much larger than the radius of the Calabi-Yau three-fold, implying that there is a large energy range where an effective 5-d description of the physics applies [27]. The bulk effective action takes the form

$$
S=\frac{M_{5}}{2} \int_{\mathcal{M}_{5}} d^{5} x \sqrt{-g}\left(R-\frac{1}{2}(\partial \phi)^{2}-\frac{3}{2} \frac{e^{2 \phi} \mathcal{F}^{2}}{5 !}\right)
$$

where the scalar modulus field $\phi$ represents the radius of the Calabi-Yau three-fold, $\mathcal{F}$ is the field strength of a four-form gauge field, and the rest of the notation is standard. Note that the dilaton is assumed to be frozen.

There are two orbifold fixed planes, the visible brane located at $y=0$, and the hidden brane located at $y=R$, where $y$ is the coordinate in the orbifold direction, and $R$ is the orbifold radius. Associated with each orbifold fixed plane is a brane action, whose form will not be important in the following. Important for the following discussion is the fact that several fields are present in the bulk, setting the stage for the possible generation of entropy fluctuations.

The above action admits a symmetric BPS solution which contains, in addition to the orbifold fixed planes, a bulk brane parallel to the boundary fixed planes, located at the position $y=Y$. The solution takes the form [1], 4] (generalized from [27])

$$
\begin{aligned}
d s^{2} & \left.=D(y)\left(-N^{2} d \tau^{2}+A^{2} d \mathbf{x}^{2}\right)+B^{2} D^{4}(y) d y^{2}\right) \\
e^{\phi} & =B D^{3}(y) \\
\mathcal{F}_{0123 y} & =-A^{3} N B^{-1} \alpha D^{-2}(y)(y<Y) \\
& =-A^{3} N B^{-1}(\alpha-\beta) D^{-2}(y)(y>Y),
\end{aligned}
$$

where $\tau$ is conformal time and

$$
\begin{aligned}
D(y) & =\alpha y+C(y<Y) \\
& =(\alpha-\beta) y+C+\beta Y \quad(y>Y),
\end{aligned}
$$

state, with all degrees of freedom, including winding strings 23] and branes [25], excited. This brane gas scenario has the potential of explaining why there are only three large spatial dimensions, but it does not (yet) provide a mechanism of structure formation.

${ }^{\S}$ However, this alone does not explain a second aspect of the "flatness problem" which is naturally solved by inflation, namely why the spatial size of the visible brane is large enough to agree with present observations, or, equivalently, why the entropy is so large 4 . 
with $A, B, C, N$ and $Y$ being constants and $C>0$. The associated tensions of the visible, hidden and bulk branes are $-\alpha M_{5}^{3},(\alpha-\beta) M_{5}^{3}$ and $\beta M_{5}^{3}$, respectively, where both $\alpha$ and $\beta$ are positive.

The action (11) does not include non-perturbative interactions between the bulk and boundary branes. Such are assumed to lead to a potential $V(Y)$ with which the bulk brane is attracted to the visible brane. If the bulk brane is light and the interaction forces are weak, then the dynamics of a configuration starting out in the BPS state (2) corresponds to the bulk brane moving adiabatically towards the visible brane. This dynamics can be described [1] in terms of a moduli space action in which the parameters $A, B, C, N$ and $Y$ of the ansatz (2) are taken to be time dependent. The effective action for these parameters is obtained by inserting the ansatz (2) into the action (11) and integrating over space. If $\mathbf{x}$ dependence of the parameters is to be allowed (which we must when studying fluctuations), then only integration over the orbifold direction $y$ is performed. In either case, the potential $V(Y)$ must be added to the effective Lagrangian by hand. In [1], the parameters $B$ and $C$ were left to be constant, yielding an 4-d effective action for a single "scalar field" $Y$ in a cosmological background with scale factor $a$ determined by $A$ and $N$.

In the Ekpyrotic Universe scenario, branes and bulk are assumed to start out in the cold, symmetric BPS state given by (2). It is assumed that the light bulk brane is nucleated near the hidden brane, and that it then slowly moves towards the visible brane, attracted by the potential $V(Y)$. Associated with the bulk brane is another brane action. After integrating over the orbifold direction, this action yields a four-dimensional effective action for a scalar field $\varphi$ related to the orbifold position $Y$. The potential produced by nonperturbative effects in string theory was taken to be (see e.g. [28] for some work on the origin of such potentials)

$$
V(\varphi)=-V_{0} e^{-\sqrt{\frac{2}{p}}\left(m_{p}\right)^{-1} \varphi}
$$

where $0<p \ll 1$ and $m_{p}$ denotes the 4 -d Planck mass (using the notation of [13]).

The proposed mechanism of structure formation is based on quantum vacuum fluctuations in the position of the bulk brane **. In the 4-d effective field theory description, these fluctuations are represented as perturbations of the scalar field $\varphi$. The analysis of fluctuations performed in [1, 1, 13 was based on considering the 4-d effective action for gravity in the presence of the scalar field $\varphi$. In [1], the time delay formalism of 12 was used to determine the fluctuations in the time of impact of the bulk brane on the visible brane, which in turn determines the resulting density fluctuations. The result is

\footnotetext{
** The initial classical fluctuations must be tuned to be smaller than the induced quantum vacuum perturbations [4].
}

a scale-invariant spectrum of adiabatic fluctuations. The time delay method, however, is not accurate when the expansion of space is not quasi-exponential [29], as in this case and similar to power law inflation. Lyth [13], on the other hand, focused on $\zeta$, the curvature perturbation in comoving coordinates 30] (denoted by $-\mathcal{R}$ in [13] - we follow the notation of [31]), a quantity which is conserved on scales larger than the Hubble radius in the absence of non-adiabatic perturbations [30,32 34]. He showed that because $\zeta$ is conserved the curvature fluctuations remain small in amplitude and have a blue spectrum, as they do in the pre-big-bang models which only take into account the dilaton field (Lyth's calculations confirm that in the absence of metric fluctuations the power spectrum of fluctuations in $\varphi$ is scale-invariant).

However, from the form of the BPS solution (2) it is clear that fluctuations in the position of the bulk brane do not decouple from fluctuations in other fields such as the modulus field $\Phi$. Including the dynamics of these fields may give a different dynamics on which the fluctuations evolve. Neglecting these coupling means eliminating the isocurvature modes which are inevitably present in the Ekpyrotic Universe and which will be discussed in a followup paper. These latter allow for the establishment and growth of entropy fluctuations on super-Hubble scales, in close analogy to what occurs in the case of axion fluctuations in inflationary cosmology (see e.g. [19]) and in the case of the growth of fluctuations on superHubble scales during the reheating period of inflationary cosmology 35,36 (such growth does not occur in single field models [36]37], but it does occur in the case of the existence of unsuppressed entropic fluctuations [38,39]).

\section{THE ORIGINAL EFFECTIVE FIELD THEORY FOR THE EKPYROTIC UNIVERSE}

The effective action for fluctuations used in [1] and 13] is

$$
S=\int d^{4} x \sqrt{-g}\left(\frac{1}{2 \kappa^{2}} R+\frac{1}{2}(\partial \varphi)^{2}-V(\varphi)\right),
$$

where, as mentioned before, $\varphi$ is a scalar field which represents the position of the bulk brane in the fifth dimension, and where $\kappa^{2}=m_{p}^{-2}=8 \pi G$.In this effective field theory, fluctuations in the position of the bulk brane generate adiabatic curvature perturbations.

The background equations of motion relevant to the following analysis are

$$
\ddot{\varphi}+3 H \dot{\varphi}+V^{\prime}=0
$$

and

$$
H^{2}=\frac{1}{3 m_{p}^{2}}\left(\frac{1}{2} \dot{\varphi}^{2}+V(\varphi)\right),
$$

where an overdot denotes the derivative with respect to physical time, the prime in $V^{\prime}$ denotes the derivative with respect to $\varphi$, and $H$ is the effective Hubble constant. 
The relevant solution of (6, 17) is given by

$$
a(t) \propto(-t)^{p}
$$

or

$$
H=\frac{p}{t} .
$$

In this solution, the time $t$ is negative. Since

$$
m_{p}^{2} \dot{H}=-\frac{1}{2} \dot{\varphi}^{2},
$$

we can determine the solution for $\dot{\varphi}$

$$
\dot{\varphi}^{2}=\frac{2 p m_{p}^{2}}{t^{2}}
$$

from which the background values of all relevant quantities can be determined. In particular,

$$
\begin{aligned}
V & =-(1-3 p) p \frac{m_{p}^{2}}{t^{2}} \\
V^{\prime} & =\sqrt{2 p}(1-3 p) \frac{m_{p}}{t^{2}} .
\end{aligned}
$$

In order to determine the evolution of the curvature fluctuations, we make use of the gauge-invariant formalism of [31] and focus on the gravitational potential $\Phi$ which appears in the metric which includes linear fluctuations (in longitudinal gauge) as

$$
d s^{2}=a^{2}(\eta)\left[(1+2 \Phi) d \eta^{2}-(1-2 \Phi) d x^{i} d x_{i}\right],
$$

where $\eta$ is conformal time. By inserting this metric and the decomposition of the scalar field $\varphi$ into background and fluctuation $\delta \varphi$, it was shown by Mukhanov [40] (see also [41]) that the following combination $v$ of metric and matter fluctuations corresponds to the canonically normalized fluctuation variable

$$
v=a\left(\delta \varphi+\frac{\dot{\varphi}}{H} \Phi\right) \equiv a Q .
$$

In the absence of entropy fluctuations (e.g. in the single matter field model considered in this section), the equations of motion of fluctuations reduce to

$$
\begin{aligned}
v_{k}^{\prime \prime}+ & \left(k^{2}-\frac{a^{\prime \prime}}{a}+a^{2} V^{\prime \prime}\right. \\
& \left.+2 a^{2}\left[\frac{\dot{H}}{H}+3 H\right]^{\circ}\right) v_{k}=0,
\end{aligned}
$$

where primes on functions of time denote the derivative with respect to conformal time, and the primes on functions of $\varphi$ denote derivatives with respect to $\varphi$. Overdots denote derivatives with respect to physical time.

From the background equations of motion it follows that the last two terms on the left hand side of (16) cancel out. The only remaining term in the frequency for $v$ is therefore the curvature, i.e. $a^{\prime \prime} / a$ :

$$
\frac{a^{\prime \prime}}{a}=p \frac{2 p-1}{(p-1)^{2} \eta^{2}} .
$$

The solution for $Q$ normalized by the initial vacuum state condition can thus be expressed in terms of Hankel functions $H_{\nu}$ :

$$
Q_{k}=A(-\eta)^{\nu} H_{\nu}^{(1)}(-k \eta)
$$

where the index $\nu$ and amplitude $A$ are given by

$$
\nu=\frac{1}{2} \frac{1-3 p}{1-p}, \quad A=e^{i\left(\nu+\frac{1}{2}\right) \frac{\pi}{2}} \frac{\sqrt{\pi}}{2} \frac{1}{(1-p)^{\frac{p}{1-p}}} .
$$

The above solution agrees with the Minkowski limit for $-k \eta \rightarrow \infty$. For long wavelengths $(-k \eta \rightarrow 0), Q$ scales as 433:

$$
Q_{k} \simeq A(-\eta)^{\nu}\left[\frac{(-k \eta / 2)^{\nu}}{\Gamma(\nu+1)}-i \frac{\Gamma(\nu)}{\pi(-k \eta / 2)^{\nu}}\right] .
$$

The curvature perturbation $\zeta$ introduced by Bardeen [30] is related to $Q$ as follows:

$$
\zeta=\frac{H}{\dot{\phi}} Q .
$$

By using the behavior for long wavelengths (20) and inserting the background values for $H$ and $\dot{\varphi}$, one obtains for the spectrum of curvature perturbations:

$$
\begin{aligned}
P_{\zeta}(k) & =\frac{k^{3}}{2 \pi^{2}}\left|\zeta_{k}\right|^{2} \\
& =\frac{p k^{3-2 \nu}}{4 m_{p}^{2} \pi^{4}}|A|^{2} 2^{2 \nu} \Gamma(\nu)^{2} .
\end{aligned}
$$

Note that the spectrum is independent of time, a wellknown result 32 34, and that its amplitude is proportional to $p$.

For extremely slow contraction [1], i.e. $p \sim 0$ or equivalently $\nu \sim 1 / 2$, one obtains a blue spectrum of curvature perturbations with index $n \sim 3$ and with an amplitude which is suppressed by the small number $p$, in agreement with the result of Lyth. The reason for this is the crucial effect due to metric perturbations in Eq. (16). The bulk brane fluctuations in rigid spacetime, i.e. without the inclusion of metric perturbations, are amplified by the negative effective mass [1, [1] and obtain a scale invariant spectrum. However, when one include self-consistently the metric perturbations, the instability due to the negative effective mass is exactly cancelled by the gravitational contribution $\boxplus$, and the bulk brane fluctuations

\footnotetext{
${ }^{\dagger \dagger}$ A similar exact cancellation between the potential term and the gravitational corrections also occurs in power-law inflation 42].
} 
satisfy the same equation of a massless minimally coupled scalar field. If contraction is very slow, the curvature term (17) in Eq. (16) is very inefficient in amplifying fluctuations and $v$ (or $Q$ ) essentially remains in its initial vacuum state. In principle a scale invariant spectrum for $Q$ can be obtained for $p=2 / 3$ (not constant in time) or for $p>>1$ (constant in time). In the latter case it is not clear if such a fast contraction can successfully solve the horizon problem.

On the other hand (as pointed out in [44]), the generalized Newtonian (Bardeen) potential $\Phi$ does grow during the Ekpyrotic phase. The general solution for $\Phi$ on scales much larger than the Hubble radius is 31 .

$$
\Phi=S \frac{H}{a}+D\left(\frac{1}{a} \int a d t\right)
$$

where $S$ and $D$ are constants. In an expanding Universe, e.g. in inflationary cosmology, $S$ is the coefficient of the decaying mode, and $D$ is the coefficient of the dominant mode. In a contracting background, e.g. in the preimpact phase of the Ekpyrotic Universe or in the prebounce phase of pre-big-bang cosmology, the S-mode is a growing mode. For a scale factor described by a power of time, the D-mode is constant. This is the case both in an expanding and in a contracting background.

In our case the scale factor is approximately constant (it decays with a very small power of $\eta$ ) the growing mode scales as $\eta^{-1}$. The growth starts at the time $\eta_{k}$ when the scale labelled by $k$ exits the Hubble radius

$$
\eta_{k} \propto \frac{1}{k}
$$

Hence, the spectrum of the growing mode of $\Phi$ is scaleinvariant

$$
\begin{aligned}
P_{\Phi}(k, \eta) & =\frac{k^{3}}{2 \pi^{2}}\left|\Phi_{k}(\eta)\right|^{2} \\
& =\frac{k^{3}}{2 \pi^{2}}\left(\frac{\eta_{k}}{\eta}\right)^{2}\left|\Phi_{k}\left(\eta_{k}\right)\right|^{2} \\
& \propto k^{0}
\end{aligned}
$$

Note that the spectrum of the D-mode of $\Phi$ does not obtain the factor $\eta_{k}^{2}$ and hence is blue $(n=3)$.

The results of the two previous paragraphs can be obtained from our earlier equations concerning $\zeta$ by relating the generalized Newtonian potential to the time variation of $\zeta$ in the usual way [36]:

$$
k^{2} \Phi_{k}=-\frac{1}{2 m_{p}^{2}} \frac{\varphi^{\prime 2}}{\mathcal{H}} \zeta_{k}^{\prime}
$$

By using

$$
\zeta_{k}^{\prime}=\sqrt{\frac{p}{2}} \frac{k A}{m_{p}}(-\eta)^{\nu} H_{\nu-1}^{(1)}(-k \eta)
$$

and Eq. (26) one obtains for the Newtonian potential for small $k$ :

$$
\Phi_{k}=-\frac{A \sqrt{2 p}}{m_{p}(1-p)}\left[\frac{(-\eta)^{2 \nu-2}}{2^{\nu} \Gamma(\nu) k^{2-\nu}}-i \frac{2^{\nu-2} \Gamma(\nu-1)}{\pi k^{\nu}}\right]
$$

From this equation it is clear that $\Phi$ obtains a component constant in time $\sim k^{-\nu}$ and a growing mode with spectrum $\sim k^{\nu-2}$. This latter component for $p \sim 0$ gives rise to a scale-invariant spectrum.

The natural question which we are going to address in the next section is the fate of this growing mode. Of course, such growing modes in contracting universes are physical. The gauge is completely specified in Eq. (14) and thus $\Phi$ for nonzero $k$ has a gauge-invariant meaning. Since $\Phi$ - and not only $\zeta$ - enters in observables such as the CMB anisotropies without any suppression factors, its spectrum leaves a direct imprint. Therefore, the only way that the growing mode of $\Phi$ in the contracting phase can disappear from physical observables at late times is if this mode only matches to the decaying mode of the post-impact radiation-dominated era. We will show that this is exactly what occurs.

A similar effect arises in the pre-big-bang scenario. Also in that context, the growing mode of the contracting phase of the pre-big-bang phase does not match to the constant mode of the standard big-bang phase 17 (It is not an issue of "gauging away" the growing mode as stated in [15]).

\section{MATCHING CONDITIONS}

As we have seen in the previous section, the growing mode of the Bardeen potential $\Phi$ is increasing during the Ekpyrotic phase and obtains a scale-invariant spectrum, whereas the curvature parameter $\zeta$ does not grow and is not scale-invariant (it has $n=3$ ). This situation is similar to what occurs in pre-big-bang cosmology [15]. During the radiation dominated post-impact phase, both $\zeta$ and the non-decaying mode of $\Phi$ are constant. Lyth [13] argues that $\zeta$ is continuous across the transition and that hence the final curvature fluctuations are not scale invariant and have negligible amplitude. However, since the variable $\Phi$ enters directly in the expression for the cosmic microwave anisotropies, one could argue that the scale-invariance of the growing mode of $\Phi$ will lead to scale-invariance of late time cosmological observables (this is the claim made in [44]).

In the following we will use the Hwang-Vishniac [16] and Deruelle-Mukhanov 17 matching conditions for cosmological fluctuations to demonstrate that the preimpact growing mode of $\Phi$ does not couple to the postimpact dominant (i.e. non-decaying) mode of $\Phi$. Since the subdominant (constant) mode of $\Phi$ during the preimpact phase is not scale-invariant, this implies that the spectrum of $\Phi$ at late times is determined by the initial spectrum of the constant mode of $\Phi$, and that it is hence neither large in amplitude nor scale-invariant, thus confirming the results of Lyth. 
The Deruelle-Mukhanov matching conditions which relate the fluctuations across a discontinuous transition in the equation of state are applicable if the transition occurs on a surface of constant energy density. This is satisfied in the pre-big-bang scenario since (presumably) the graceful exit mechanism which determines the bounce occurs at some fixed energy density. In the Ekpyrotic scenario, the transition surface is determined by when the bulk brane impacts on the visible brane. Since the energy of the visible brane before impact is constant in space and time (given by the brane tension) and since for long-wavelength fluctuations the extra energy density imparted to the visible brane by the collision with the bulk brane is independent of space when measured at the time of impact, the above condition for applicability of the Deruelle-Mukhanov matching conditions is satisfied. The matching conditions imply that both $\Phi$ and

$$
\hat{\zeta}=\zeta-\frac{1}{3} k^{2} \Phi\left(\mathcal{H}^{\prime}-\mathcal{H}^{2}\right)^{-1}
$$

are continuous across the transition. For large-scale fluctuations, this implies the continuity of $\Phi$ and $\zeta$. Since the total energy density does not change at the impact time, we take the magnitude of $\mathcal{H}$ to be continuous across the transition 周

We write the generalized Newtonian potential $\Phi$ before impact as

$$
\Phi_{-}=D_{-}+S_{-} \frac{\mathcal{H}_{-}}{a^{2}}
$$

and after impact as

$$
\Phi_{+}=D_{+}+S_{+} \frac{\mathcal{H}_{+}}{a^{2}} .
$$

Here, the coefficients $D_{i}(i= \pm)$ denote the amplitudes of the constant modes, $S_{-}$is the amplitude of the growing mode during the Ekpyrotic phase, and $S_{+}$is that of the decaying mode after the Ekpyrotic phase. Subscripts - and + denote the limiting values of the quantities evaluated at the impact time on the pre-impact and post-impact branches.

We find $D_{+}, S_{+}$as a function of $D_{-}, S_{-}$by matching $\Phi$ and $\hat{\zeta}$.It is useful to recall the expression for $\zeta$ in terms of $\Phi[31]$ :

$$
\zeta=\frac{2}{3} \frac{\Phi+H^{-1} \dot{\Phi}}{1+w}+\Phi
$$

\footnotetext{
${ }^{\ddagger \ddagger}$ Note that we are matching a contracting phase of cosmological evolution to an expanding phase at the impact time, similar to what is done in the Einstein frame description of Pre-Big-Bang cosmology. The impact in the model of [⿰亻⿱⺊口灬] curs at a surface of finite density.
}

Setting $k=0$ leads to the following system of equations:

$$
\begin{aligned}
& D_{+}=D_{-}+\frac{\mathcal{H}_{-} S_{-}-\mathcal{H}_{+} S_{+}}{a^{2}} \\
& \frac{\mathcal{H}_{-}}{\mathcal{H}_{-}^{\prime}-\mathcal{H}_{-}^{2}}\left(\mathcal{H}_{-} D_{-}+\frac{S_{-}}{a^{2}}\left(\mathcal{H}_{-}^{\prime}-\mathcal{H}_{-}^{2}\right)\right) \\
= & \frac{\mathcal{H}_{+}}{\mathcal{H}_{+}^{\prime}-\mathcal{H}_{+}^{2}}\left(\mathcal{H}_{+} D_{+}+\frac{S_{+}}{a^{2}}\left(\mathcal{H}_{+}^{\prime}-\mathcal{H}_{+}^{2}\right)\right)
\end{aligned}
$$

The second equation can be rewritten as:

$$
\frac{\mathcal{H}_{+}^{2}}{\mathcal{H}_{+}^{\prime}-\mathcal{H}_{+}^{2}} D_{+}=\frac{\mathcal{H}_{-}^{2}}{\mathcal{H}_{-}^{\prime}-\mathcal{H}_{-}^{2}} D_{-}+\frac{S_{-} \mathcal{H}_{-}-S_{+} \mathcal{H}_{+}}{a^{2}} .
$$

We subtract Eq. (33) from Eq. (35) and we get:

$$
\frac{\mathcal{H}_{+}^{\prime}-2 \mathcal{H}_{+}^{2}}{\mathcal{H}_{+}^{\prime}-\mathcal{H}_{+}^{2}} D_{+}=\frac{\mathcal{H}_{-}^{\prime}-2 \mathcal{H}_{-}^{2}}{\mathcal{H}_{-}^{\prime}-\mathcal{H}_{-}^{2}} D_{-} .
$$

Thus, the late-time constant mode receives no contribution from the pre-impact increasing mode. Hence, the final amplitude of $\Phi$ is small, and its spectrum is not scale-invariant. This result agrees with the conclusions of Deruelle and Mukhanov in the case of the pre-big-bang theory. Note that in the case of an inflationary Universe modeled with an equation of state with $w$ constant during and after inflation, but with a discontinuous change in the equation of state at the end of the period of inflation, then Eq. (36) reproduces the well-known result 32, 33 that the value of $\Phi$ increases by the ratio of the factors $1+w$ between the period of inflation and the radiation-dominated phase.

If we now take into account the term in $\zeta$ proportional to $k^{2}$, then Eq. (35) becomes

$$
\begin{gathered}
\frac{1}{\mathcal{H}_{-}^{\prime}-\mathcal{H}_{-}^{2}}\left(\left(\mathcal{H}_{-}^{2}+\frac{k^{2}}{3}\right) D_{-}+\frac{\mathcal{H}_{-} S_{-}}{a^{2}}\left(\mathcal{H}_{-}^{\prime}-\mathcal{H}_{-}^{2}+\frac{k^{2}}{3}\right)\right)= \\
\frac{1}{\mathcal{H}_{+}^{\prime}-\mathcal{H}_{+}^{2}}\left(\left(\mathcal{H}_{+}+\frac{k^{2}}{3}\right) D_{+}+\frac{\mathcal{H}_{+} \mathcal{S}_{+}}{a^{2}}\left(\mathcal{H}_{+}^{\prime}-\mathcal{H}_{+}^{2}+\frac{k^{2}}{3}\right)\right) .
\end{gathered}
$$

This change leads to

$$
D_{+}=A D_{-}+B k^{2}\left(S_{+}-S_{-}\right)
$$

which means that the imprint of the growing mode during the contracting phase on the constant mode of the standard big bang phase is suppressed by $k^{2}$ and is therefore unimportant in the long wavelength limit for $p \sim 0$. However, we note that for $p \sim 2 / 3$ (for this case $\zeta$ has a scale invariant spectrum) there is the possibility to get a scale invariant spectrum for $\Phi$ in the expanding phase by the matching with the growing mode in the contracting phase (the growing mode is $\Phi \sim\left(k^{7 / 2} \eta^{5}\right)^{-1}$ ).

\section{DISCUSSION}

Perhaps the most important aspect of the proposed Ekpyrotic Universe scenario is the claim that it gives rise 
to a mechanism to produce a scale-invariant spectrum of adiabatic cosmological fluctuations with an amplitude which can be tuned to agree with observations.

We have studied a four-dimensional toy model of the Ekpyrotic scenario. In this context, we have used the Hwang-Vishniac [16] and Deruelle-Mukhanov [17] matching conditions to demonstrate that there is indeed a growing mode of the adiabatic metric fluctuations (quantified most conveniently in terms of the generalized Newtonian potential $\Phi$ during the pre-impact phase, and that this mode has a scale-invariant spectrum (in agreement with the conclusions of [44]), but that this mode does not couple to the non-decaying mode of the post-impact phase. The non-growing mode of $\Phi$ during the pre-impact phase does have a blue spectrum $(n=3)$. The final spectrum of fluctuations in the radiation-dominated phase is thus blue and of negligible amplitude. These conclusions can also be obtained by focusing on the variable $\zeta$. Our work confirms that $\zeta$ is indeed constant across the transition, (as argued in [13,45]). We warn the reader that our conclusions are based on the toy model studied, and may change in a calculation which better encodes the higherdimensional stringy aspects of the Ekpyrotic scenario.

Note that our approach to this four-dimensional toy model of the Ekpyrotic Universe assumes that the matching happens at a finite value of the effective fourdimensional scale factor, as suggested in [1]. It is also based on using the negative potential at the impact point, whereas in [1] it is suggested that the potential should vanish close to $Y=0$, as has been emphasized in 44 . However, this modification of the potential alone is unlikely to yield a scale-invariant spectrum. It makes the model look more like the Pre-Big-Bang scenario, and also yields a blue spectrum.

Our matching conditions applied to a toy model of inflation with a jump of the equation of state at reheating (as opposed to a continuous change), but with constant equation of state before and after reheating, give the correct result 16, 17], whereas the matching proposed in [44], namely the continuous matching of the two modes of the fluctuation variable $\epsilon_{m}$ (the energy density contrast on comoving hypersurfaces, which is directly related to $\Phi$ by a function of the background scale factor) does not. In a new version of the Ekpyrotic Universe [50] published after this manuscript was completed, it is proposed that the big bang of the visible Universe results from the collision of the two boundary branes, at which point the four-dimensional scale factor vanishes. In this case any four-dimensional toy model will break down completely and matching fluctuations at this singular point seems hazardous.

Another caveat is that this four-dimensional effective theory is (as emphasized in [7] ) not consistent since it involves a sudden transition between contraction and expansion which is not consistent with the background Einstein equations. However, we think that by adopting the matching conditions chosen here, spurious effects due to this sudden transition are avoided. Further- more, there are many effects concerning fluctuations in the five-dimensional bulk which cannot be captured in a four-dimensional effective theory of perturbations. The final word concerning the spectrum of fluctuations in the Ekpyrotic Universe scenario will have to come from a full 5 -d analysis of the fluctuation equations, using e.g. the formalism recently proposed in 4649]. These calculations, in turn, will have to be based on a consistent 5-d analysis of the background dynamics.

\section{Acknowledgments}

This research was supported in part by the U.S. Department of Energy under Contracts DE-FG0291ER40688, TASK A (Brown Univ.) and DE-FG0291ER40681, TASK B (Purdue Univ.). Both authors thank Salman Habib for hospitality at the 2001 Summer Workshop on Cosmology during which some of this work was done. One of us (F.F.) is grateful to Raul Abramo for useful comments. R.B. thanks David Lyth and Paul Steinhardt for communicating to him early drafts of their unpublished papers on this subject, and Andrei Linde and David Lyth for important feedback on an early set of notes. He is grateful to Nathalie Deruelle, Jai-chan Hwang, David Wands and in particular Neil Turok for fruitful discussions on the issues presented here, and Lev Kofman and Burt Ovrut for feedback on the manuscript. He thanks the organizers of the M-theory cosmology conference in Cambridge for the opportunity to debate these issues, the Department of Physics and Astronomy of the University of British Columbia, and in particular Bill Unruh, for hospitality during the time this paper was written, and the UBC theory postdocs Emil Akhmedov, Sumati Surya and Kostya Zarembo for encouragement.

[1] J. Khoury, B. A. Ovrut, P. J. Steinhardt and N. Turok, "The ekpyrotic universe: Colliding branes and the origin of the hot big bang," hep-th/0103239.

[2] P. Horava and E. Witten, "Heterotic and type I string dynamics from eleven dimensions," Nucl. Phys. B 460, 506 (1996) hep-th/9510209.

[3] A. H. Guth, "The Inflationary Universe: A Possible Solution To The Horizon And Flatness Problems," Phys. Rev. D 23, 347 (1981).

[4] R. Kallosh, L. Kofman and A. Linde, "Pyrotechnic universe," hep-th/0104073.

[5] J. Khoury, B. A. Ovrut, P. J. Steinhardt and N. Turok, "A brief comment on 'The pyrotechnic universe'," hepth/0105212

[6] R. Y. Donagi, J. Khoury, B. A. Ovrut, P. J. Steinhardt and N. Turok, "Visible branes with negative tension in heterotic M-theory," hep-th/0105199.

[7] R. Kallosh, L. Kofman, A. Linde and A. Tseytlin, "BPS branes in cosmology," hep-th/0106241. 
[8] K. Enqvist, E. Keski-Vakkuri and S. Rasanen, "Hubble Law and Brane Matter after Ekpyrosis," hep-th/0106282.

[9] N. Turok, "A Causal Source which Mimics Inflation," Phys. Rev. Lett. 77, 4138 (1996) astro-ph/9607109].

[10] P. P. Avelino and C. J. Martins, "Primordial adiabatic fluctuations from cosmic defects," Phys. Rev. Lett. 85, 1370 (2000) astro-ph/0002413.

[11] E. J. Copeland, R. Easther and D. Wands, "Vacuum fluctuations in axion-dilaton cosmologies," Phys. Rev. D 56, 874 (1997) hep-th/9701082.

[12] A. H. Guth and S. Y. Pi, "Fluctuations in the New Inflationary Universe," Phys. Rev. Lett. 49, 1110 (1982).

[13] D. H. Lyth, "The primordial curvature perturbation in the ekpyrotic universe," hep-ph/0106153.

[14] M. Gasperini and G. Veneziano, "Pre - big bang in string cosmology," Astropart. Phys. 1, 317 (1993) hepth/9211021.

[15] R. Brustein, M. Gasperini, M. Giovannini, V. F. Mukhanov and G. Veneziano, "Metric perturbations in dilaton driven inflation," Phys. Rev. D 51, 6744 (1995) hep-th/9501066.

[16] J. Hwang and E. T. Vishniac, "Gauge-Invariant Joining Conditions for Cosmological Perturbations," Astrophs. J. 382, 363 (1991).

[17] N. Deruelle and V. F. Mukhanov, "On matching conditions for cosmological perturbations," Phys. Rev. D 52, 5549 (1995) gr-qc/9503050.

[18] J. Martin and D. J. Schwarz, "The influence of cosmological transitions on the evolution of density perturbations," Phys. Rev. D 57, 3302 (1998) gr-qc/9704049.

[19] M. Axenides, R. Brandenberger and M. Turner, "Development of Axion Perturbations in an Axion Dominated Universe," Phys. Lett. B 126, 178 (1983).

[20] A. D. Linde, "Generation Of Isothermal Density Perturbations In The Inflationary Universe," JETP Lett. 40, 1333 (1984) [Pisma Zh. Eksp. Teor. Fiz. 40, 496 (1984)].

[21] D. A. Easson, "The interface of cosmology with string and M(illennium) theory," hep-th/0003086.

[22] R. H. Brandenberger, "The promise of string cosmology," hep-th/0103156.

[23] R. Brandenberger and C. Vafa, "Superstrings in the Early Universe," Nucl. Phys. B 316, 391 (1989).

[24] J. E. Lidsey, D. Wands and E. J. Copeland, "Superstring Cosmology," Phys. Rept. 337, 343 (2000).

[25] S. Alexander, R. Brandenberger and D. Easson, "Brane gases in the early universe," Phys. Rev. D 62, 103509 (2000) hep-th/0005212.

[26] A. Buonanno, T. Damour and G. Veneziano, "Pre-big bang bubbles from the gravitational instability of generic string vacua," Nucl. Phys. B 543, 275 (1999) hepth/9806230.

[27] A. Lukas, B. A. Ovrut and D. Waldram, "On the fourdimensional effective action of strongly coupled heterotic string theory," Nucl. Phys. B 532, 43 (1998) hepth/9710208;

A. Lukas, B. A. Ovrut, K. S. Stelle and D. Waldram, "The universe as a domain wall," Phys. Rev. D 59, 086001 (1999) hep-th/9803235.

[28] G. Moore, G. Peradze and N. Saulina, "Instabilities in heterotic M-theory induced by open membrane instan- tons," hep-th/0012104.

[29] L. Wang, V. F. Mukhanov and P. J. Steinhardt, "On the problem of predicting inflationary perturbations," Phys. Lett. B 414, 18 (1997) astro-ph/9709032.

[30] J. M. Bardeen, "Gauge Invariant Cosmological Perturbations," Phys. Rev. D 22, 1882 (1980).

[31] V. F. Mukhanov, H. A. Feldman and R. H. Brandenberger, "Theory of cosmological perturbations," Phys. Rept. 215, 203 (1992).

[32] J. M. Bardeen, P. J. Steinhardt and M. S. Turner, "Spontaneous Creation of Almost Scale - Free Density Perturbations in an Inflationary Universe," Phys. Rev. D 28, 679 (1983).

[33] R. Brandenberger and R. Kahn, "Cosmological Perturbations in Inflationary Universe Models," Phys. Rev. D 29, 2172 (1984).

[34] D. H. Lyth, "Large Scale Energy Density Perturbations and Inflation," Phys. Rev. D 31, 1792 (1985).

[35] B. A. Bassett, D. I. Kaiser and R. Maartens, "General relativistic preheating after inflation," Phys. Lett. B 455, 84 (1999) hep-ph/9808404.

[36] F. Finelli and R. Brandenberger, "Parametric amplification of gravitational fluctuations during reheating," Phys. Rev. Lett. 82, 1362 (1999) hep-ph/9809490.

[37] M. Parry and R. Easther, "Preheating and the Einstein field equations," Phys. Rev. D 59, 061301 (1999) [hepph/9809574.

[38] B. A. Bassett and F. Viniegra, "Massless metric preheating," Phys. Rev. D 62, 043507 (2000) hep-ph/9909353.

[39] F. Finelli and R. Brandenberger, "Parametric amplification of metric fluctuations during reheating in two field models," Phys. Rev. D 62, 083502 (2000) hhep$\mathrm{ph} / 0003172$.

[40] V. F. Mukhanov, "Quantum Theory Of Gauge Invariant Cosmological Perturbations," Sov. Phys. JETP 67, 1297 (1988) [Zh. Eksp. Teor. Fiz. 94N7, 1 (1988)].

[41] M. Sasaki, "Large Scale Quantum Fluctuations In The Inflationary Universe," Prog. Theor. Phys. 76, 1036 (1986).

[42] D. H. Lyth and E. D. Stewart, "The Curvature Perturbation in Power Law (e.g. Extended) Inflation", Phys. Lett. B 274, 168 (1992).

[43] M. Abramowicz and I. A. Stegun, Handbook of Mathematical Functions (Dover, New York, 1972).

[44] J. Khoury, B. Ovrut, P. Steinhardt and N. Turok, "Gravitational backreaction and density perturbations in ekpyrotic models", to be submitted.

[45] D. Lyth, "The curvature perturbation in the ekpyrotic and pre-big-bang Universes", to be submitted.

[46] C. van de Bruck, M. Dorca, R. H. Brandenberger and A. Lukas, "Cosmological perturbations in brane-world theories: Formalism," Phys. Rev. D 62, 123515 (2000) hep-th/0005032.

[47] R. Maartens, "Cosmological dynamics on the brane," Phys. Rev. D 62, 084023 (2000) hep-th/0004166.

[48] D. Langlois, "Brane cosmological perturbations," Phys. Rev. D 62, 126012 (2000) hep-th/0005025.

[49] H. Kodama, A. Ishibashi and O. Seto, "Brane world cosmology: Gauge-invariant formalism for perturbation," Phys. Rev. D 62, 064022 (2000) hep-th/0004160. 
[50] J. Khoury, B. A. Ovrut, N. Seiberg, P. J. Steinhardt and N. Turok, "From Big Crunch To Big Bang", hepth/0108187. 